\title{
Towards an understating of signal transduction protein interaction networks
}

\author{
Dowluru SVGK Kaladhar ${ }^{*}$, Potladurthi Chandra Sai ${ }^{2}$, Padmanabhuni V Nageswara Rao1, \\ Amajala Krishna chaitanya1, Duddukuri Govinda Rao1, Vadlapudi Varahala Rao ${ }^{3}$, Erva \\ Rajeswara Reddy², Sudabattula Vijaya kumar ${ }^{2}$ \& Divyakolu Vinod Kumar1
}

${ }^{1}$ GITAM University, Visakhapatam; ${ }^{2}$ Madanapalle Institute of Technology and Science, Madanapalle, Chittor; ${ }^{3}$ Dr Lankapalli Bullayya post graduate college, Visakhapatnam; DSVGK Kaladhar - E-mail: dr.dowluru@gmail.com; *Corresponding author

Received April 11, 2012; Accepted April 27, 2012; Published May 15, 2012

\begin{abstract}
:
Protein network analysis has witnessed a number of advancements in the past for understanding molecular characteristics for important network topologies in biological systems. The signaling pathway regulates cell cycle progression and anti-apoptotic molecules. This pathway is also involved in maintaining cell survival by modulating the activity of apoptosis through RAS, P13K, $\mathrm{AKT}$ and BAD activities. The importance of protein-protein interactions to improve usability of the interactome by scoring and ranking interaction data for proteins in signal transduction networks is illustrated using available data and resources.
\end{abstract}

Keywords: Signal transduction, Apoptosis, Angiogenesis

\section{Background:}

Intracellular communication or environmental signals in eukaryotic cells responds with growth adaptation or cell death requires reconstruction of specific patterns of gene expression [1]. The external signals are transduced to intracellular effectors by pathways that link cell surface and nuclear events and processed transient input to permanent responses [2].

Genes such as Ras are activated by point mutation through transduction. Others such as Src were identified as the homologous of transforming viral genes. The development of computer algorithms to predict optimal intervention is a future development in Ras/Raf/MAPK Kinase cascade [3]. The impact of genomic and proteomic technologies on the development of new cancer drugs evaluated by using metabolic pathways is known. Li et al., (2011) proposed biological methods to analyze the roles of ERK1/2 signaling pathways in regenerating hepatosites using Bioinformatics tools [4]. Various metabolic pathways such as KEGG are being organized to computerized functional aspects of genes and genomics in terms of molecular interactions and gene relationships. Various metabolic databases is being composed based on three interconnected sessions; genes, molecules and pathways [5].
Angiogenesis is the growth of new blood vessels which occurs during both physiological and pathological disease processes [6]. Cancer cells can spread to other parts of the body through blood and lymphatic systems [7]. Protein-protein interaction forms the basis for majority of cellular events such as signal transduction, transcriptional regulation and gene regulation in various living organisms. Here, we describe the importance of protein-protein interactions in protein networks.

\section{Methodology:}

System requirement

System requirements are Intel® Core TM i3 CPU M 370 @ 2.40 $\mathrm{GHz} 2.40 \mathrm{GHz}$. Processor, 4.00 GB (3.80 usable) RAM, LENOVO Base Mother Board, 500 GB Hard disk, Windows 7 Professional (x64) (build 7600) OS.

\section{Pathway data and source}

KEGG stands for Kyoto Encyclopedia of Genes and Genomes and it is one of the most important, manually constructed, constantly updated metabolic pathway database available online for free. The database describes pathways for signal transductions, primary and secondary metabolic cycles, and immune systems. We used data from KEGG for this study. 


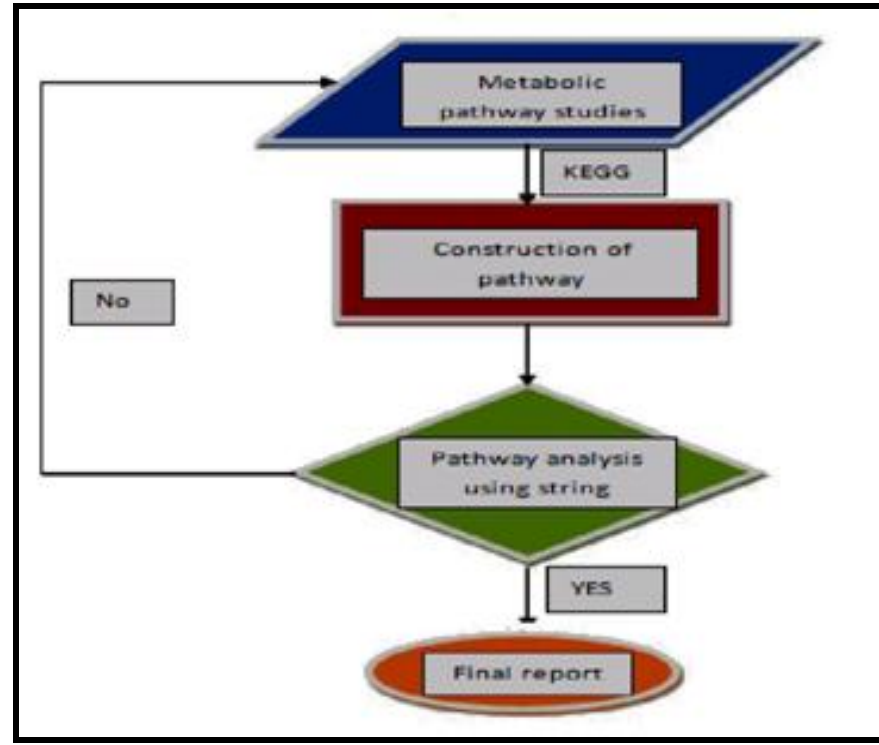

Figure 1: Flow chart for the workflow analysis used in this study; KEGG = Encyclopedia of Genes and Genomes

\section{Protein interaction data and tool}

We used STRING (version 9.0) for extracting protein-protein interaction data. STRING is a database of experimentally known and predicted protein-protein interactions. The interactions include direct (physical) and indirect (functional) associations. They are derived from four sources: (1) genomic context; (2) high-throughput experiments; (3) co-expression (conserved) and (4) known knowledge. STRING quantitatively integrates interaction data from these sources for a large number of organisms, and transfers information between these organisms where applicable. The procedure used in this study for combining, excluding and comparing data in KEGG and STRING is illustrated using a flowchart in (Figure 1).

\section{Discussion:}

The RAS/RAF/MEK/ERK signaling pathway regulates cell cycle progression and anti-apoptotic molecules. Mutations are often observed among molecules in the pathway in transformed cell lines and human cancer cells. This pathway is also involved in maintaining cell survival by modulating the activity of apoptosis including RAS, P13K, AKT and BAD. This is illustrated using (Figure 2). The mechanisms in the complex network of factors contributing to human diseases is often been analyzed using data extracted from KEGG. The active process of cell suicide termed "programmed cell death" is initiated either by injury to the cell due to exogenous damaging agents or by changes in endogenous signals by RAS/P13K/AKT/BAD. Androgens are capable of stimulating proliferation and imbalance in program cell death due to RAS/RAF/MEK/ERK occurs during several cancer progressions. Coordination of cell proliferation with antiapoptotic signals results in activation of receptor thyrosine and serine/threonine protein kinases leading to RAS action (Figure 2).

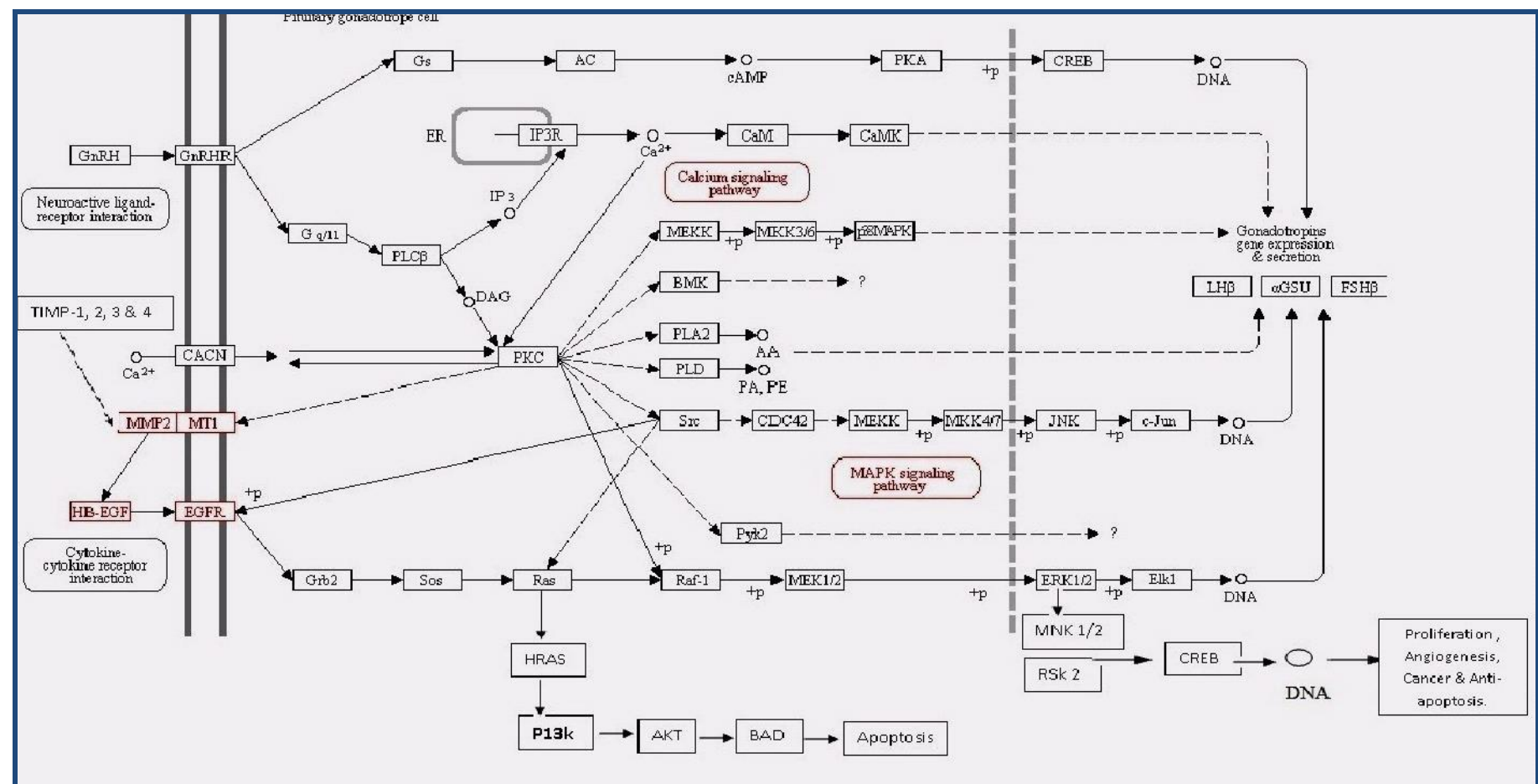

Figure 2: A pathway describing cell proliferation with anti-apoptotic signals with the activation of receptor thyrosine and serine/threonine protein kinases is illustrated

A protein interaction network summarizes a large amount of protein-protein interaction data to reveal aspects of network topology and modularity. Due to increasingly large amount of heterogeneous raw data becoming available, proper data integration is essential for protein network reconstruction.
This is true especially in the development of human protein interaction networks. Several algorithms aim at clustering proteins into complex networks with biological functions using functional genomics and Bioinformatics analysis derived data. 


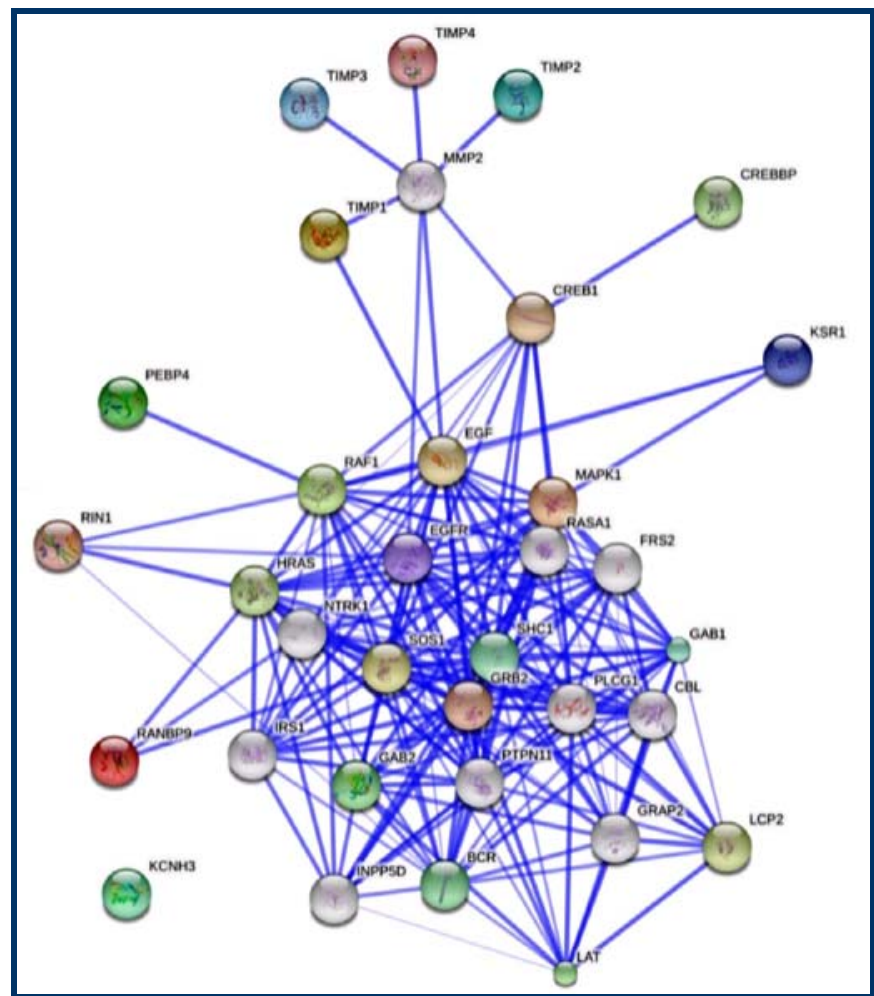

Figure 3: Example of a protein interaction network from STRING (version 9.0) is shown for signal transduction associated proteins.

The interaction data in STRING (version 9.0) include physical interactions as well as functional associations predicted in common pathway or process derived using knowledge from DB such as BIND, KEGG, HPRD and REACTOM. STRING aims to improve usability of the interactome by scoring and ranking interaction data. (Figure 3) illustrates interaction of PKCMT1MMP2/EGFR/GRB2/SOS/RAS/RAF/MEK/ERK/ RSK/CREB and PKC/MT1MMP2/EGFR/GRB2/SOS/RAS/P13K/AKT/ BAD networks using STRING data.

The MAPK pathway is the heart of molecular signaling networks for human (with about 22,400 genes). This governs growth, proliferation, differentiation and survival. This is also associated with mutations linked to cancer, Alzheimer's and Parkinson's diseases [8]. The current survey illustrates the complex, less understood interactive pathway with genes responsible for apoptotic and anti-apoptotic actions. Munshi $e t$ al. (2004) provided that transforming growth factor $\beta 1$ (TGF$\beta 1$ ) regulates a wide array of cellular process including cell differentiation, proliferation and apoptosis [9].

An efficient genetic system to predict protein-protein interaction between two proteins with known or unknown protein shapes and structures is critical [10]. The contribution by residues from both proteins to energy (thermodynamics and kinetics) is now possible to be measured by double mutant cycle analysis. This may help in the better understanding of protein networks and protein-protein interactions associated with activated EGFR involving metallo-proteins leading to apoptosis and anti-apoptosis.

Christian et al. (1999) [13] proposed macro molecular associations with representations using metabolic networks (KEGG) of Human Genetic Disease (OMIM) data. The HPRD DB provides protein names and their synonym to help in such complex networks. Hence, the available clinical, biochemical and bioinformatics approaches help delineate the possible function of enzymes/proteins by generating clues in bacteria, virus, plants and animals [11, 12]. Thus, the problem of protein-protein interactions in a biological system is of fundamental importance in understanding implicit and explicit information related to proteins in networks [13].

\section{Conclusion:}

The importance of protein-protein interactions and their predictions in the development of complex protein networks such as the signal transduction is highlighted.

\section{Competing interests:}

The authors declare that they have no competing interests.

\section{Acknowledgment:}

The authors acknowledge the support from GITAM University for providing the necessary research facilities.

\section{References:}

[1] Benoit P et al. J Biol Chem. 1998 273: 29661 [PMID: 9792677]

[2] Kuang E et al. J Biol Chem. 2009 284: 13958 [PMID: 19304659]

[3] Workman P, Ann Oncol. 2002 13: 115 [PMID: 12401677]

[4] Li M et al .J Genet. 2011 90: e82-5. [PMID: 22232197]

[5] Goto S et al. Pac Symp Biocomput. 1997 175: 86 [PMID: 9390290]

[6] Slevin M et al. J Angiogenes Res. 2009 1: 1. [PMID: 19946409]

[7] Sethi JK \& Vidal-Puig A, J Biochem. 2010 427: 1 [PMID: 20226003]

[8] Orton RJ et al. Biochem. J. 2005 392: 249 [PMID: 16293107]

[9] Munshi HG et al. J Biol Chem. 2004 279: 39042 [PMID: 15247230]

[10] Jones S \& Thorton JM, Proc Natl Acad Sci USA. 1996 93: 13 [PMID: 8552589]

[11] Sridhar GR et al. Med Hypotheses. 2010 75: 648 [PMID: 20797821]

[12] Kaladhar DSVGK, IJPSRR. 2011 137: 141

[13] Iseli C et al. Proc Int Conf Intell Syst Mol Biol. 1999 138: 48 [PMID: 10786296]
Edited by $P$ Kangueane

Citation: Kaladhar et al. Bioinformation 8(9): 437-439 (2012)
restricted use, distribution, and reproduction in any medium, License statement: This is an open-access article, which permits unrestricted use, distribution, and reproduction in any medium,
for non-commercial purposes, provided the original author and source are credited 